\title{
Analysis of the expression of SDF-1 splicing variants in human colorectal cancer and normal mucosa tissues
}

\author{
RISALA HUSSAIN ALLAMI ${ }^{1 *}$, CLAUDINE GRAF $^{2 *}$, KSENIA MARTCHENKO $^{3}$, BEATRICE VOSS $^{2}$, \\ MARC BECKER ${ }^{1}$, MARTIN R. BERGER ${ }^{4}$, PETER R. GALLE ${ }^{1}$, MATTHIAS THEOBALD ${ }^{2}$, \\ THOMAS C. WEHLER ${ }^{2 * *}$ and CARL C. SCHIMANSKI ${ }^{3 * *}$ \\ ${ }^{1}$ First Department of Internal Medicine; ${ }^{2}$ Third Department of Internal Medicine, \\ Johannes Gutenberg University Hospital of Mainz, D-55130 Mainz; ${ }^{3}$ Department of Internal Medicine, \\ Marienhospital Darmstadt, D-64285 Darmstadt; ${ }^{4}$ Research Group Toxicology and Chemotherapy, \\ German Cancer Research Center, D-69117 Heidelberg, Germany
}

Received May 28, 2014; Accepted June 22, 2015

DOI: $10.3892 / 01.2016 .4139$

\begin{abstract}
C-X-C motif chemokine ligand 12 (CXCL12), also termed stromal cell-derived factor-1 (SDF-1) is a small protein 8-14 $\mathrm{kDa}$ in length that is expressed as six isoforms, consisting

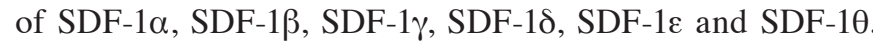
All six isoforms are encoded by the single CXCL12 gene on chromosome 10. This gene regulates leukocyte trafficking and is variably expressed in a number of normal and cancer tissues. The potential role of the novel CXCL12 splice variants as components of the CXCR4 axis in cancer development is not fully understood. The present study aimed to analyze the expression profile of the various SDF-1 isoforms and SDF-1 polymorphisms, and the association with the clinicopathological features and overall survival of patients with colorectal cancer (CRC). SDF-1 polymorphism analysis was performed using restriction fragment length polymorphism (RFLP) analysis in 73 histologically confirmed human CRC tissue samples at various stages of disease. The expression pattern of the SDF-1 isoforms was analyzed by reverse transcription-polymerase chain reaction in 40 histologically confirmed human CRC tissue samples obtained at various stages of disease, as well as in matched adjacent normal mucosa samples. The presence of the CXCL12 gene polymorphism rs1801157 demonstrated an association with local progression of the primary tumor, as indicated by the $\mathrm{T}$ stage. The frequency of the GG genotype was slightly increased in patients with stage 3 and 4 tumors (78.0\%) compared with
\end{abstract}

Correspondence to: Dr Ksenia Martchenko, Department of Internal Medicine, Marienhospital Darmstadt, Martinspfad 72, D-64285 Darmstadt, Germany

E-mail:k.martchenko@marienhospital-darmstadt.de

"Contributed equally; ** Joint senior authorship

Key words: colorectal cancer, C-X-C motif chemokine ligand 12, stromal cell derived factor 1 splice variants, isoforms the incidence of the GA/AA genotype $(69.5 \%$; $\mathrm{P}=0.067)$. The expression of SDF-1 $\beta$ was associated with the presence of metastases $(\mathrm{P}=0.0656)$ and the expression of SDF- $1 \gamma$ was significantly associated with tumor size $(\mathrm{P}=0.0423)$. The present study is the first to analyze the association between the expression profile of the chemokine CXCL12 splice variants in human CRC tissues and their clinical relevance. The present results reveal that the CXCL12 G801A polymorphism is a low-penetrance risk factor for the development of $\mathrm{CRC}$, and was associated with the T stage. All six isoforms of SDF-1 were expressed in CRC tissues. The expression of SDF- $1 \beta$ was found to be associated with metastases and SDF- $1 \gamma$ appears to be a possible tumor marker for local tumor progression.

\section{Introduction}

Colorectal cancer (CRC) is one of the most frequent cancers in Western countries, and the disease is delineated by local recurrence and lymphatic and hematogenous dissemination $(1,2)$. The results of in vivo and in vitro studies performed in various tumor entities indicate that tumor progression and organ-specific metastasis is partially affected by interactions between chemokine receptors on cancer cells and the corresponding chemokines expressed in target organs. Therefore, chemokine receptors direct the spread of tumor cells and affect the sites of metastatic growth (3). Chemokines and the corresponding G-protein-coupled receptors have previously been reported to mediate various immunoresponses (4). The high expression of stromal cell derived factor 1 (SDF-1), also termed $\mathrm{C}-\mathrm{X}-\mathrm{C}$ motif chemokine ligand 12 (CXCL12), in endothelial cells, biliary epithelial cells, bone marrow stromal cells and lymph nodes results in a chemotactic gradient that attracts lymphocytes expressing $\mathrm{C}-\mathrm{X}-\mathrm{C}$ chemokine receptor type 4 (CXCR4) to the respective organs (4-6).

CXCR4 has become a focus of studies, as it is the most common chemokine receptor expressed on cancer cells (3). CXCR4 has been indicated to play an important role in tumor dissemination in colorectal, breast and oral squamous cell carcinoma, as these tumors all commonly metastasize 
to SDF-1-expressing organs $(7,8)$. Previous studies that analyzed the metastatic ability of CXCR4-expressing cancer cells using murine tumor models underlined the key role of CXCR4 in tumor cell malignancy (9-13). The activation of CXCR4 by SDF-1 $\alpha$ has been reported to induce the migration, invasion and angiogenesis of cancer cells (14-17). SDF-1 proteins are found as monomers in living organisms. Six isoforms of SDF-1 exist, consisting of SDF-1 $\alpha$, SDF-1 $\beta$, SDF-1 $\gamma$, SDF-1 $\delta$, SDF-1e and SDF-1 $\theta$, due to alternative splicing. All isoforms possess the same initial three exons and then differ from exon 4 onwards $(18,19)$. The best-known isoforms are SDF- $1 \alpha$ and SDF-1 $\beta$. The functional diversity and differential proteolytic processing properties of these two isoforms has been extensively investigated and characterized $(20,21)$. However, little is known about the other isoforms.

In the present study, the expression of the various SDF-1 isoforms and SDF-1 polymorphisms in tissue specimens obtained from CRC patients was analyzed. These results were assessed for associations between the clinicopathological parameters and overall survival of the patients.

\section{Materials and methods}

Tissue samples. CRC tissue samples were intraoperatively obtained from 73 patients, according to the ethical committee of the State Chamber of Medicine in Rhineland-Palatinate, and were derived from 30 (41\%) females and $43(59 \%)$ males, with a median age at diagnosis of 66.5 years. Written informed consent was obtained from all patients. The morphological classification of the carcinoma was performed according to World Health Organization (WHO) specifications for the tumor-node-metastasis (TNM) classification. The patients were followed up on a regular basis, depending on the procedure performed.

Restriction fragment length polymorphism (RFLP) assay. The presence of a polymorphism at codon 801 of the SDF-1 gene was analyzed by RFLP analysis using cDNA obtained from 73 human $\mathrm{CRC}$ tissue specimens. Polymerase chain reaction (PCR) was performed as described by Dimberg et al (22), using the 5'-CAGTCAACC TGGGCAAAGCC-3' and 5'-AGC TTTGGTCCTGAGAGTCC-3' primers (Eurofins Genomics, Ebersberg, Germany). The resulting double-stranded DNA was then digested using the restriction enzyme $M s p I$. To visualize the resulting DNA fragments, agarose gel electrophoresis was performed (Fig. 1).

Analysis of CXCL12 splice variant expression in human $C R C$ and normal mucosa tissues by reverse transcription $(R T)-P C R$. The expression of the CXCL12 splice variants SDF-1 $\alpha$, SDF-1 $\beta$, SDF-1 $\gamma$, SDF-1 $\delta$, SDF-1 1 and SDF-1 $\theta$ was analyzed in $40 \mathrm{CRC}$ and 20 human mucosa cDNA samples obtained from a subgroup of the aforementioned tissue samples. In order to determine the expression patterns of the human SDF-1 isoforms in CRC and normal mucosal tissues, PCR primers (Eurofins Genomics) that specifically detected each SDF-1 isoform were designed as follows: SDF-1 $\alpha$ forward, 5'-ATGAACGCCAAGGTCGTGGTC-3' and reverse, 5'-AAGTGCTTACTTGTTTAAAGCTTTCTC-3;
SDF-1 $\beta$ forward, 5'-ATGAACGCCAAGGTCGTGGTC-3' and reverse, 5'-ACCCTCTCACATCTTGAACCTCTT-3'; SDF-1 $\gamma$ forward, 5'-ATGAACGCCAAGGTCGTGGTC-3' and reverse, 5'-AGATAACTAGTTT TTCCTTT T CTGGGC-3'; SDF-1ס forward, 5'-ATGAACGCCAAGGTCGTGGTC-3' and reverse, 5'-ACACCATTACAC ATCCCCAGGAGA-3'; SDF-1 $\varepsilon$ forward, 5'-CGCCATGAACGCCAAGGTCGTGGTCG-3' and reverse, 5'-CTAATTAC TTCAGTGGCAGATCATAC-3'; and SDF-10 forward, 5'-CGCCATGAACGCCAAGGTCGTGGTCG-3' and reverse, 5'-CACACTAATTACTTCAGTGGCAGATC-3'. $\beta$-actin was used as an internal control for the tissue samples (23). The PCR reactions were performed using an automated thermal cycler (PTC-200; Bio Rad Laboratories GmbH, Munich, Germany).

Statistical analysis. The association between the SDF-1 G801A polymorphism or isoform expression and the clinicopathological features of patients was assessed using box plots and the Wilcoxon test if the explanatory variable had 2 levels, or the Kruskal-Wallis test if there were $>2$ levels of the explanatory variable. The association between the patient age, $\beta$-actin expression and isoform expression was determined using Pearson product-moment correlation coefficient and reported as scatterplot matrices. $\mathrm{P} \leq 0.05$ was considered to indicate a statistical significant difference. Statistical analysis was performed using SAS 9.2 software from SAS Institute, Inc. (Cary, NC, USA).

\section{Results}

Association between SDF-1 isoforms and clinicopathological parameters. The expression of the CXCL12 splice variants SDF- $1 \alpha$, SDF-1 $\beta$, SDF- $1 \gamma$, SDF-1 $\delta$, SDF- $1 \varepsilon$, and SDF-1 10 was evaluated in 40 established CRC and 20 normal mucosa tissue specimens. RT-PCR analysis revealed that the mRNA of these SDF-1 splice variants was expressed in CRC and normal mucosa tissues (Table I; Fig. 1). The expression rates of the SDF- $1 \alpha$, SDF-1 $\beta$, SDF- $1 \gamma$, SDF-1 $\delta$, SDF- $1 \varepsilon$ and SDF-1 10 splice variants were $80,70,50,37.5,30$ or $32.5 \%$, respectively. By contrast, the expression rates of the SDF-1 $\alpha$, SDF-1 $\beta$, SDF-1 $\gamma$, SDF-1 $\delta$, SDF-1 $1 \varepsilon$ or SDF-1 $\theta$ splice variants in the normal mucosa were $65,50,25,40,45$ and $35 \%$, respectively (23).

In total, 40 tumors obtained from 39 patients were included in the present study. For 1 patient, clinical data was not available. Out of the total 39 patients, 22 patients $(56 \%)$ were male. The analyzed tumors were located in the caecum in 3 patients $(8 \%)$, colon ascendens in 5 patients $(13 \%)$, colon transversum in 1 patient $(3 \%)$, colon descendens in 2 patients $(5 \%)$, sigma in 13 patients $(33 \%)$ and rectum in 15 patients (38\%). The tumors were stage T3 in 29 patients (74\%), whereas 2 patients possessed T1 tumors (5\%), 5 patients possessed T2 tumors (13\%) and 3 patients possessed T4 tumors (8\%).

The median patient age was 65.7 years (standard deviation, 10.7), with a range of $41-83$ years. There was little association between the categorical clinical parameters, such as gender, tumor site or TNM stage and the expression of the SDF-1 isoforms, with the exception of two associations, consisting of SDF-1 $\beta$ being associated with the presence of 
Table I. Expression of SDF-1 isoforms in CRC and normal mucosa tissues.

\begin{tabular}{lcc}
\hline Isoform & $\begin{array}{c}\text { Expression of SDF-1 isoforms } \\
\text { in CRC tissue samples, } \mathrm{n}(\%)\end{array}$ & $\begin{array}{c}\text { Expression of SDF-1 isoforms in } \\
\text { normal mucosa tissue samples, } \mathrm{n}(\%)\end{array}$ \\
\hline Total & $40(100.0)$ & $20(100.0)$ \\
SDF-1 $\alpha$ & $32(80.0)$ & $13(65.0)$ \\
SDF-1 $\beta$ & $28(70.0)$ & $10(50.0)$ \\
SDF-1 $\gamma$ & $20(50.0)$ & $5(25.0)$ \\
SDF-1 $\delta$ & $15(37.5)$ & $8(40.0)$ \\
SDF-1 $\varepsilon$ & $12(30.0)$ & $9(45.0)$ \\
SDF-1 $\theta$ & $13(32.5)$ & $7(35.0)$ \\
\hline
\end{tabular}

SDF-1, stromal cell derived factor 1; CRC, colorectal cancer.

Table II. Tumour characteristics in patients expressing the CXCL12 genotypes.

\begin{tabular}{|c|c|c|c|}
\hline \multirow[b]{2}{*}{ Characteristics } & \multicolumn{2}{|c|}{ CXCL12 genotype } & \multirow[b]{2}{*}{ P-value } \\
\hline & GG, n (\%) & GA or AA, n (\%) & \\
\hline Total & $50(100.0)$ & $23(100.0)$ & \\
\hline Median age, years & 66 & 67 & 0.035 \\
\hline \multicolumn{4}{|l|}{ Gender } \\
\hline Male & $27(54.0)$ & $16(69.6)$ & 0.579 \\
\hline Female & $23(46.0)$ & $7(30.5)$ & NS \\
\hline \multicolumn{4}{|l|}{ T stage } \\
\hline $1+2$ & $11(22.0)$ & $7(30.5)$ & 0.067 \\
\hline $3+4$ & $39(78.0)$ & $16(69.5)$ & \\
\hline \multicolumn{4}{|l|}{$\mathrm{N}$ stage } \\
\hline 0 & $12(24.0)$ & $6(26.0)$ & 0.364 \\
\hline $1-3$ & $37(76.0)$ & $17(74.0)$ & NS \\
\hline \multicolumn{4}{|l|}{ M stage } \\
\hline 0 & $33(66.0)$ & $13(56.5)$ & 0.509 \\
\hline+ & $17(34.0)$ & $10(43.5)$ & NS \\
\hline \multicolumn{4}{|l|}{ R status } \\
\hline 0 & $32(64.0)$ & $11(47.8)$ & 0.395 \\
\hline+ & $18(36.0)$ & $12(52.2)$ & NS \\
\hline \multicolumn{4}{|l|}{ T stage } \\
\hline $1-3$ & $39(78.0)$ & $13(56.5)$ & 0.214 \\
\hline 4 & $11(22.0)$ & $10(43.5)$ & NS \\
\hline
\end{tabular}

$\mathrm{N}$-status could not be obtain from one patient. CXCL12, C-X-C motif chemokine ligand 12; NS, not significant; T stage, tumor stage; N stage, stage of lymph node involvement; $\mathrm{M}$ stage, metastasis stage; R status, residual tumor status.

distant metastases $(\mathrm{P}=0.0656)$ and $\mathrm{SDF}-1 \gamma$ being significantly positively associated with tumor size $(\mathrm{P}=0.0423)(23)$.

Genotype frequency. The CXCL12 splice variants were successfully detected in all patient samples. The frequency of the GG CXCL12 allele was compared with the frequency of the GA and AA CXCL12 alleles for the patient gender, patient age and clinical presentation (Table II). Out of the 73 patients with CRC, GG homozygosity was observed in 50 patients with
CRC (68.5\%), whereas 23 CRC patients possessed the GA and AA genotypes (31.5\%; Fig. 2). With the exception of the local progression of the primary tumor, no significant associations were identified between the rs1801157 CXCL12 gene polymorphism and the clinicopathological parameters. The rs1801157 CXCL12 gene polymorphism was associated with the local progression of the primary tumor, as indicated by the $\mathrm{T}$ stage. The frequency of the GG genotype was notably increased in patients with stage 3 and 4 tumors (78.0\%) 


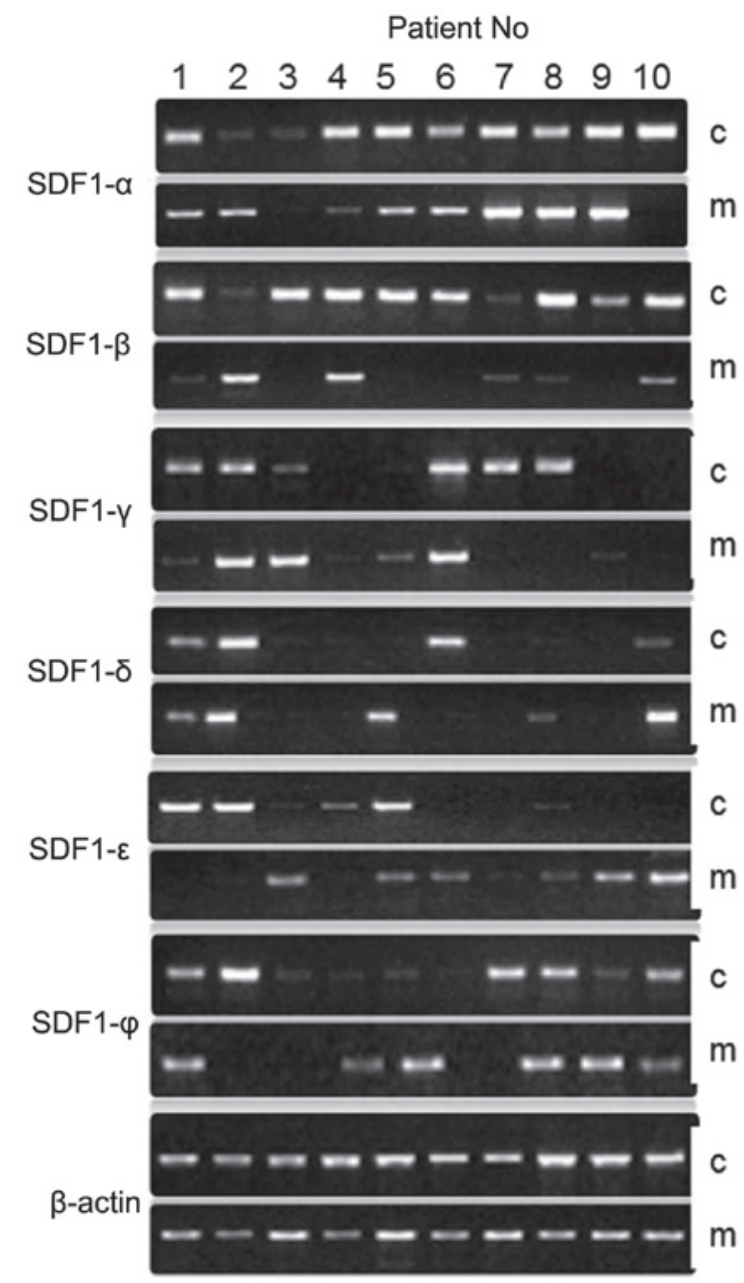

Figure 1. Expression of SDF-1 isoforms in colorectal carcinoma and human normal mucosa tissues. Polymerase chain reaction analysis of the SDF-1 isoforms in cDNA samples obtained from colorectal carcinoma tissues and matched normal mucosa tissues adjacent to the tumor. The experiments were repeated twice and identical results were obtained. SDF-1, stromal cell derived factor $1 ; \mathrm{c}$, colorectal carcinoma tissue; $\mathrm{m}$, normal mucosa tissue.

compared with the incidence of the GA or AA genotypes $(69.5 \%$; $=0.067)(23)$.

\section{Discussion}

SDF-1 is considered to be important for the pathogenesis of certain diseases $(24,25)$. The known CXCL12 isoforms, SDF-1 $\alpha$, SDF-1 $\beta$, SDF- $1 \gamma$, SDF-1 $\delta$, SDF- $1 \varepsilon$ and SDF-1 $\theta$ result from alternative splicing events of the same gene (26). In addition, a single-nucleotide polymorphism (SNP), termed CXCL12-G801A or rs1801157, was identified in the 3 -untranslated region of the CXCL12 gene transcript.

Several studies have examined the association between disease susceptibility and the CXCL12-G801A polymorphism(27-33). These studies indicated that the CXCL12-G801A polymorphism was associated with an increased risk of HIV infection, lung cancer, breast cancer, acute leukemia, prostate cancer, non-Hodgkin's lymphoma and oral squamous cell carcinoma (27-34). However, only a small number of studies focused on the presence of the CXCL12-G801A polymorphism in CRC patients to clarify whether the CXCL12-G801A
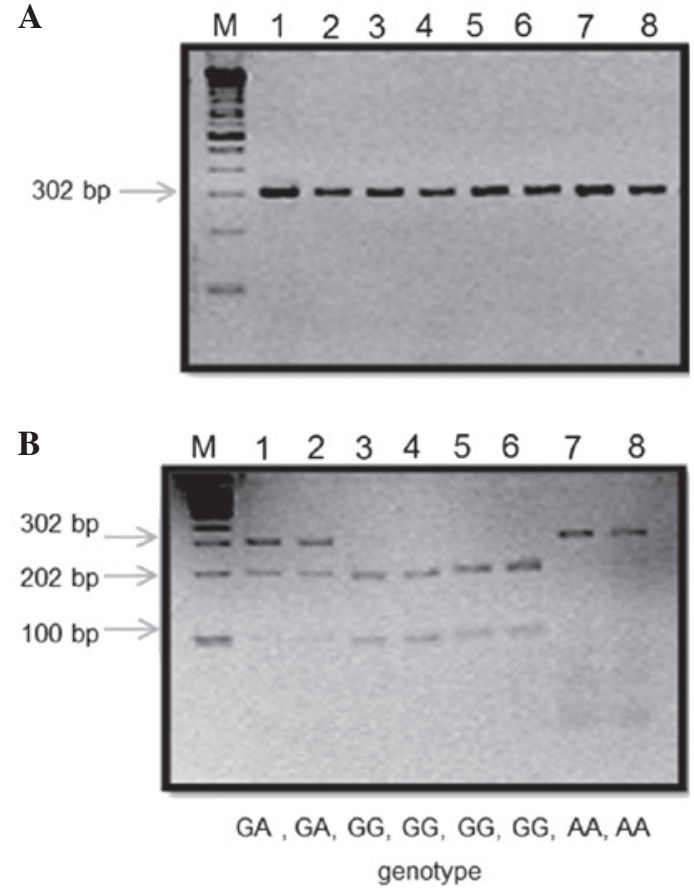

Figure 2. (A) Polymerase chain reaction products for CXCL12 from representative CRC patients. Lane M, molecular marker (100 bp). (B) Restriction fragment length polymorphism analysis of CXCL12 gene polymorphism in representative CRC patients. Lane $\mathrm{M}$, molecular marker (100bp); lanes 1 and 2, mutated heterozygote GA genotype (100, 202 and $302 \mathrm{bp}$ ); lanes 3-6, wild type homozygous GG genotype (100 and 202 bp); lanes 7 and 8, mutated homozygous AA genotype (302 bp). CXCL12, C-X-C motif chemokine ligand 12; CRC, colorectal cancer.

polymorphism is associated with disease susceptibility and clinical characteristics in CRC $(31,35)$.

A total of 73 patients were included in the present study, and the $\mathrm{G} / \mathrm{G}$ polymorphism was identified in 50 patients with CRC (68.5\%), and the $\mathrm{G} / \mathrm{A}$ and $\mathrm{A} / \mathrm{A}$ genotypes were identified in 23 patients with CRC (31.5\%). Comparison of the results that were obtained in the present analysis of the CXCL12 gene polymorphism in human CRC with results obtained previously is of importance. The results of the present study were consistent with the results of Dimberg et al (22), who studied the CXCL12 gene polymorphism in 151 patients with CRC and 141 control individuals. This study reported that the frequency of the G/G, G/A and A/A genotypes were 55.6\% (84/151), $41.1 \%$ $(62 / 151)$ and $3.3 \%(5 / 151)$, respectively, in the patients with CRC. The genotype distribution was not significantly different between the CRC patients and control individuals (22).

The present study attempted to determine the association between the presence of the CXCL12 gene polymorphism and the development of CRC. The data in the present study revealed that the GG genotype distribution demonstrates a strong association with the local progression of CRC.

Previous studies have reported that the effect of the CXCL12 gene polymorphism may contribute differentially to CRC by mediating tumor progression, angiogenesis, metastasis and leukocyte migration (22).

Dimberg et al (22) and Hidalgo-Pascual et al (36) did not identify a significant correlation between the CXCL12-AA genotype and various prognostic markers, such as the development of metastasis and disease-free survival time. The present 
data were consistent with these studies, as the current results only identified an association between this genotype and the presence of larger primary tumors at primary diagnosis.

However, the study by Chang et al (37) compared the frequency of six SDF-1 $\alpha$ SNPs in 424 patients with primary T3 stage CRC, and reported that the SDF-1 $\alpha-$ G801A polymorphism was associated with lymph node metastasis. The frequency of GA/AA genotypes was significantly increased $(54.8 \%)$ in the group of patients with lymph node metastasis compared with the group of patients without lymph node metastasis $(40.7 \%)$. In the patients without lymph node metastasis, the disease-free survival rate was decreased in patients with the GA/AA genotype (74\%) compared with patients with the GG genotype $(87.6 \%)$. This previous study concluded that the SDF-1 $\alpha-G 801 \mathrm{~A}$ polymorphism may increase the expression of SDF-1 $\alpha$ mRNA and be a predictive marker of lymph node metastasis in CRC (37). The differences observed between the studies, including the present study, are likely to be due to a difference in patient numbers and tumor stages.

To assess the strength of the association between the presence of the CXCL12-G801A polymorphism and the risk of cancer, Gong et al (38) performed a meta-analysis of 17 studies comprising a total of 3,048 cancer patients and 4522 control individuals. The odds ratio (OR) and $95 \%$ confidence interval (CI) were used to determine the association. Compared with the GG genotype, the results revealed that the variant genotypes, consisting of GA (OR, 1.38; 95\% CI, 1.18-1.61) and GA/AA (OR, $1.36 ; 95 \%$ CI, 1.17-1.59), were associated with a significantly increased risk of all cancer types. This meta-analysis indicated that the CXCL12 G801A polymorphism is a low-penetrance risk factor for cancer development $(23,38)$.

Furthermore, the present study analyzed the correlation between SDF-1 isoforms and clinicopathological parameters. It was demonstrated that SDF-1 $\beta$ was associated with the presence of metastases and that SDF- $1 \gamma$ was significantly associated with tumor size. To the best of our knowledge, the present study is the first to analyze the expression profile of the chemokine CXCL12 splice variants in a larger series of human CRC tissues and normal human mucosa.

Investigation of the expression of novel CXCL12 splice variants and understanding of the potential pathophysiological relevance of the variants under various conditions is required. Different splice variants in certain cases may exhibit similar functions but are active in different tissues or physiological conditions. An improved understanding of the functional diversity of SDF-1 splicing variants may lead to novel therapies that target these variants, which may delay or inhibit the metastatic process in cancer cells (23).

In the present study, SDF-1 $\alpha$ was the most predominant splicing variant in CRC and normal mucosa tissues, followed by SDF-1 $\beta$, whereas the other splice variants $\gamma, \delta, \varepsilon$ and $\theta$ were expressed less abundantly. The present findings are consistent with previous data from the literature $(26,39,40)$, which demonstrated that SDF-1 $\alpha$ is a ubiquitously expressed splicing variant with multiple functions in various organs. The SDF-1 $\beta$ isoform appears to be involved in inter-organ communication through blood-mediated gradients and also in the support of angiogenesis $(26,39,40)$. SDF-1 $\beta$ was mainly found in highly vascularized organs, such as the liver, spleen, bone marrow and kidneys, but was absent in the brain $(26,41,42)$.
The data obtained in the present study therefore suggested that SDF- $1 \alpha$, SDF-1 $\beta$ and SDF- $1 \gamma$ may act as tumor markers, but are not necessarily associated with the patient prognosis.

\section{References}

1. Greenlee RT, Murray T, Bolden S and Wingo PA: Cancer statistics, 2000. CA Cancer J Clin 50: 7-33, 2000.

2. Weir HK, Thun MJ, Hankey BF, Ries LA, Howe HL, Wingo PA, Jemal A, Ward E, Anderson RN and Edwards BK: Annual report to the nation on the status of cancer, 1975-2000, featuring the uses of surveillance data for cancer prevention and control. J Natl Cancer Inst 95: 1276-1299, 2003.

3. Zlotnik A: Chemokines and cancer. Int J Cancer 119: 2026-2029, 2006.

4. Premack BA and Schall TJ: Chemokine receptors: gateways to inflammation and infection. Nat Med 2: 1174-1178, 1996.

5. Bagglioni M: Chemokines and leukocyte traffic. Nature 392: 565-568, 1998.

6. Bleul CC, Schultze JL and Spinger TA: B lymphocyte chemotaxis regulated in association with microanatomic localization, differentiation state and B cell receptor engagement. J Exp Med 187: 753-762, 1998.

7. Schimanski CC, Schwald S, Simiantonaki N, Javasinghe C, et al: Effect of chemokine receptors CXCR4 and CCR7 on the metastatic behavior of human colorectal cancer. Clin Cancer Res 11: 1743-1750, 2005.

8. Hao L, Zhang C, Wang L, Luo Y, et al: Recombination of CXCR4, VEGF and MMP-9 predicting lymph node metastasis in human breast cancer. Cancer Lett 253: 34-42, 2007.

9. Shanmugam MK, Manu KA, Ong TH, Ramachandran L, Surana R, Bist P, Lim LH, Kumar AP, Hui KM and Sethi G: Inhibition of CXCR4/CXCL12 signaling axis by ursolic acid leads to suppression of metastasis in transgenic adenocarcinoma of mouse prostate model. Int J Cancer 129: 1552-1563, 2011.

10. D'Alterio C, Barbieri A, Portella L, Palma G, Polimeno M, Riccio A, Ieranò C, Franco R, Scognamiglio G, Bryce J, et al: Inhibition of stromal CXCR4 impairs development of lung metastases. Cancer Immunol Immunother 61: 1713-1720, 2012.

11. Liang Z, Yoon Y, Votaw J, Goodman MM, Williams L and Shim H: Silencing of CXCR4 blocks breast cancer metastasis. Cancer Res 65: 967-971, 2005.

12. Smith MC, Luker KE, Garbow JR, Prior JL, Jackson E, Piwnica-Worms D and Luker GD: CXCR4 regulates growth of both primary and metastatic breast cancer. Cancer Res 64: 8604-8612, 2004.

13. Phillips RJ, Burdick MD, Lutz M, Belperio JA, Keane MP and Strieter RM: The stromal derived factor-1/CXCL12-CXC chemokine receptor 4 biological axis in non-small cell lung cancer metastases. Am J Respir Crit Care Med 167: 1676-1686, 2003.

14. Sun XS, Wei L, Chen Q and Terek RM: CXCR4/SDF1 mediate hypoxia induced chondrosarcoma cell invasion through ERK signaling and increased MMP1 expression. Mol Cancer 9: 17, 2010.

15. Pan J, Mestas J, Burdick MD, Phillips RJ, Thomas GV, Reckamp K, Belperio JA and Strieter RM: Stromal derived factor-I (SDF-I/CXCL12) and CXCR4 in renal cell carcinoma metastasis. Mol Cancer 5: 56, 2006.

16. Kollmar O, Rupertus K, Scheuer C, Junker B, et al: Stromal cell-derived factor-1 promotes cell migration and tumor growth of colorectal metastasis. Neoplasia 9: 862-870, 2007.

17. Mori T, Doi R, Koizumi M, Toyoda E, et al: CXCR4 antagonist inhibits stromal cell-derived factor-1-induced migration and invasion of human pancreatic cancer. Mol Cancer Ther 3: 29-37, 2004.

18. Yu L, Cecil J, Peng SB, Schrementi J, et al: Identification and expression of novel isoforms of human stromal cell-derived factor 1. Gene 374: 174-179, 2006.

19. Janowski M: Functional diversity of SDF-1 splicing variants. Cell Adh Migr 3: 243-249, 2009.

20. Lombardi L, Tavano F, Morelli F, Latiano TP, Di Sebastiano P and Maiello E: Chemokine receptor CXCR4: Role in gastrointestinal cancer. Crit Rev Oncol Hematol 88: 696-705, 2013.

21. Chada S, Ramesh R and Mhashilkar AM: Cytokine- and chemokine-based gene therapy for cancer. Curr Opin Mol Ther 5: 463-474, 2003. 
22. Dimberg J, Hugander A, Löfgren S and Wagsater D: Polymorphism and circulating levels of the chemokine CXCL12 in colorectal cancer patients. Int J Mol Med 19: 11-15, 2007.

23. Allami RH: Influence of the chemokine CXCL12 on the progression and the signalling in colorectal cancer. $\mathrm{PhD}$ dissertation, Der Johannes Gutenberg-Universität. ArchiMed, Mainz, 2013.

24. Manetti M, Liakouli V, Fatini C, Cipriani P, Bonino C, Vettori S, Guiducci S, Montecucco C, Abbate R, Valentini G, Matucci-Cerinic M, Giacomelli R and Ibba-Manneschi L: Association between a stromal cell derived factor1 (SDF-1/cxcl12) gene polymorphism and microvascular disease in systemic sclerosis. Ann Rheum Dis 68: 408-411, 2009.

25. Liu W, Zhu E, Wang R, Wang L and Liu T: CXCL12 G801A polymorphism is associated with an increased risk of benign salivary gland tumors in the Chinese population. Med Oncol 29: 677-681, 2012.

26. Yu L, Cecil J, Peng SB, Schrementi J, Kovacevic S, Paul D, Su EW and Wang J: Identification and expression of novel isoforms of human stromal cell-derived factor 1 . Gene 374 174-179, 2006.

27. Winkler C, Modi W, Smith MW, Nelson GW, Wu X, Carrington $\mathrm{M}$, Dean $\mathrm{M}$, Honjo $\mathrm{T}$, Tashiro $\mathrm{K}$, Yabe D, Buchbinder S, Vittinghoff E, Goedert JJ, O'Brien TR, Jacobson LP, Detels R, Donfield S, Willoughby A, Gomperts E, Vlahov D, Phair J and O'Brien SJ: Genetic restriction of AIDS pathogenesis by an SDF-1 chemokine gene variant. ALIVE Study, Hemophilia Growth and Development Study (HGDS), Multicenter AIDS Cohort Study (MACS), Multicenter Hemophilia Cohort Study (MHCS), San Francisco City Cohort (SFCC) Science 279: 389-393, 1998.

28. Razmkhah M, Doroudchi M, Ghayumi SM, Erfani N and Ghaderi A: Stromal cell-derived factor-1 (SDF-1) gene and susceptibility of Iranian patients with lung cancer. Lung Cancer 49: 311-315, 2005.

29. Razmkhah M, Talei AR, Doroudchi M, Khalili-Azad T and Ghaderi A: Stromal cell-derived factor-1 (SDF-1) alleles and susceptibility to breast carcinoma. Cancer Lett 225: 261-266, 2005.

30. Dommange F, Cartron G, Espanel C, Gallay N, Domenech J, Benboubker L, Ohresser M, Colombat P, Binet C, Watier H and Herault O: GOELAMS Study Group: CXCL12 polymorphism and malignant cell dissemination/tissue infiltration in acute myeloid leukemia. Faseb J 20: 1913-1915, 2006.

31. Hirata H, Hinoda Y,Kikuno N, Kawamoto K, Dahiya AV, Suehiro Y, Tanaka Y and Dahiya R: CXCL12 G801A polymorphism is a risk factor for sporadic prostate cancer susceptibility. Clin Cancer Res 13: 5056-5062, 2007.
32. de Oliveira KB, Oda JM, Voltarelli JC, Nasser TF, Ono MA, Fujita TC, Matsuo T and Watanabe MA: CXCL12 rs1801157 polymorphism in patients with breast cancer, Hodgkin's lymphoma and non-Hodgkin's lymphoma. J Clin Lab Anal 23: 387-393, 2009.

33. Teng YH, Liu TH, Tseng HC, Chung TT, Yeh CM, Li YC, Ou YH, Lin LY, Tsai HT and Yang SF: Contribution of genetic polymorphisms of stromal cell-derived factor-1 and its receptor, CXCR4, to the susceptibility and clinicopathologic development of oral cancer. Head Neck 31: 1282-1288, 2009.

34. Vairaktaris E, Vylliotis A, Spyridonodou S, Derka S, Vassiliou S, Nkenke E, Yapijakis C, Serefoglou Z, Neukam FW and Patsouris E: A DNA polymorphism of stromal-derived factor-1 is associated with advanced stages of oral cancer. Anticancer Res 28: 271-275, 2008.

35. Zhu K, Jiang B, Hu R, Yang Y, Miao M, Li Y amd Liu Z: The CXCL12 G801A polymorphism is associated with cancer risk: A meta-analysis. PLoS One 9: e108953, 2014.

36. Hidalgo-Pascual M, Galan JJ, Chaves-Conde M, Ramírez-Armengol JA, Moreno C, Calvo E, Pelaez P, Crespo C, Ruiz A and Royo JL: Analysis of CXCL12 3'UTR G>A polymorphism in colorectal cancer. Oncol Rep 18: 1583-1587, 2007.

37. Chang SC, Lin PC, Yang SH, Wang HS, Li AF and Lin JK: SDF-lalpha G801A polymorphism predicts lymph node metastasis in stage T3 colorectal cancer. Ann Surg Oncol 16: 2323-2330, 2009.

38. Gong H, Tan M, Wang Y, Shen B, Liu Z, Zhang F, Liu Y, Qiu J, Bao E and Fan Y: The CXCL12 G801A polymorphism and cancer risk: evidence from 17 case-control studies. Gene 509: 228-231, 2012.

39. Altenburg JD, Broxmeyer HE, Jin Q, Cooper S, Basu S and Alkhatib G: A naturally occurring splice variant of CXCL12/stromal cell-derived factor 1 is a potent human immunodeficiency virus type 1 inhibitor with weak chemotaxis and cell survival activities. J Virol 81: 8140-8148, 2007.

40. Janowski M: Functional diversity of SDF-1 splicing variants. Cell Adh Migr 3: 243-249, 2009.

41. Sun X, Cheng G, Hao M, Zheng J, Zhou X, Zhang J, Taichman RS, Pienta KJ and Wang J: CXCL12/CXCR4/CXCR7 chemokine axis and cancer progression. Cancer Metastasis Rev 29: 709-722, 2010.

42. Colobran R, Pujol-Borrell R, Armengol MP and Juan M: The chemokine network II. On how polymorphisms and alternative splicing increase the number of molecular species and configure intricate patterns of disease susceptibility. Clin Exp Immunol 150: 1-12, 2007. 